01,10

\title{
Молекулярно-динамическое исследование влияния концентрации вакансий на скорость миграции границ наклона в никеле
}

\author{
(C) Г.М. Полетаев ${ }^{1}$, Р.Ю. Ракитин ${ }^{2}$ \\ ${ }^{1}$ Алтайский государственный технический университет им. И.И. Ползунова, \\ Барнаул, Россия \\ ${ }^{2}$ Алтайский государственный университет, \\ Барнаул, Россия \\ E-mail: gmpoletaev@mail.ru
}

Поступила в Редакцию 19 января 2021 г.

В окончательной редакции 19 января 2021 г.

Принята в публикации 23 января 2021 г.

Методом молекулярной динамики проведено исследование влияния концентрации вакансий на скорость миграции большеугловых границ наклона с осями разориентации $\langle 111\rangle$ и $\langle 100\rangle$ в никеле. Показано, что зависимость скорости миграции от концентрации введенных на начальном этапе моделирования вакансий имеет максимум вблизи 1\%. Падение скорости миграции при дальнейшем повышении свободного объема обусловлено преимущественно торможением границы малоподвижными вакансионными кластерами, которые при высоких концентрациях вакансий граница уже не способна сорбировать. Второй причиной снижения скорости миграции с ростом концентрации вакансий выше $1 \%$ является уменьшение поверхностного натяжения границ зерен и, соответственно, движущей силы их миграции вследствие конечной сорбционной способности границ по отношению к избыточному свободному объему.

Ключевые слова: молекулярная динамика, граница зерен, миграция, вакансия, свободный объем.

DOI: 10.21883/FTT.2021.05.50804.007

\section{1. Введение}

Миграция границ зерен в поликристаллах играет определяющую роль в процессах рекристаллизации, роста зерен, во многих фазовых превращениях. От подвижности границ в значительной степени зависит стабильность микроструктуры ультрамелкозернистых, в том числе нанокристаллических, материалов [1-3]. В настоящее время известно, что на скорость миграции границы зерен влияют несколько факторов: ориентация и тип самой границы, температура, упругие напряжения, наличие примесей или собственных дефектов кристаллической структуры [1,2]. Последние в большинстве случаев тормозят миграцию границ из-за наличия положительной энергии связи с границами [1-6], вследствие чего границы зерен, в частности, часто выступают в качестве стоков для различных дефектов и примесей. Исключением из этого правила являются вакансии, которые, согласно, например, косвенным экспериментальным данным $[7,8]$ и результатам ab initio моделирования $[9,10]$, не только не снижают скорость миграции, но и, как правило, приводят к ее росту. Это объясняется тем, что наличие дополнительного свободного объема приводит к интенсификации диффузионных перемещений атомов на границе [1,11-13].

Но в целом, влияние дополнительного свободного объема, т.е. свободного объема выше того, который характерен для идеального кристалла, на миграцию границ зерен, как отмечается в $[14,15]$, неоднозначно, поскольку границы имеют конечную емкость по отно- шению к свободному объему. Из-за этого, в частности, сокращение площади границы в результате миграции сопровождается вынужденной эмиссией избыточного свободного объема в виде вакансий обратно в кристалл, что в свою очередь снижает миграционную подвижность границы $[14,15]$.

Ввиду вышесказанного, зависимость скорости миграции границ зерен от концентрации вакансий, по всей видимости, должна быть немонотонной и иметь максимум. При экспериментальных исследованиях и первопринципном моделировании имеются сложности однозначной оценки влияния концентрации вакансий или свободного объема на скорость миграции границ. В экспериментах, как правило, является проблематичным выделение влияния только вакансий наряду с другими дефектами и локальным разогревом, возникающим вследствие внешних воздействий (облучения частицами, деформации), осуществляемых для создания высокой концентрации вакансий. Второй проблемой, общей для экспериментальных и ab initio методов, является сложность определения фактических значений концентрации вакансий и свободного объема из-за активного процесса поглощения вакансий самими границами зерен или объединения вакансий друг с другом с образованием вакансионных кластеров.

Настоящая работа посвящена исследованию влияния свободного объема на скорость миграции большеугловых границ наклона с осями разориентации $\langle 111\rangle$ и $\langle 100\rangle$ в никеле методом молекулярной динамики. Данный 

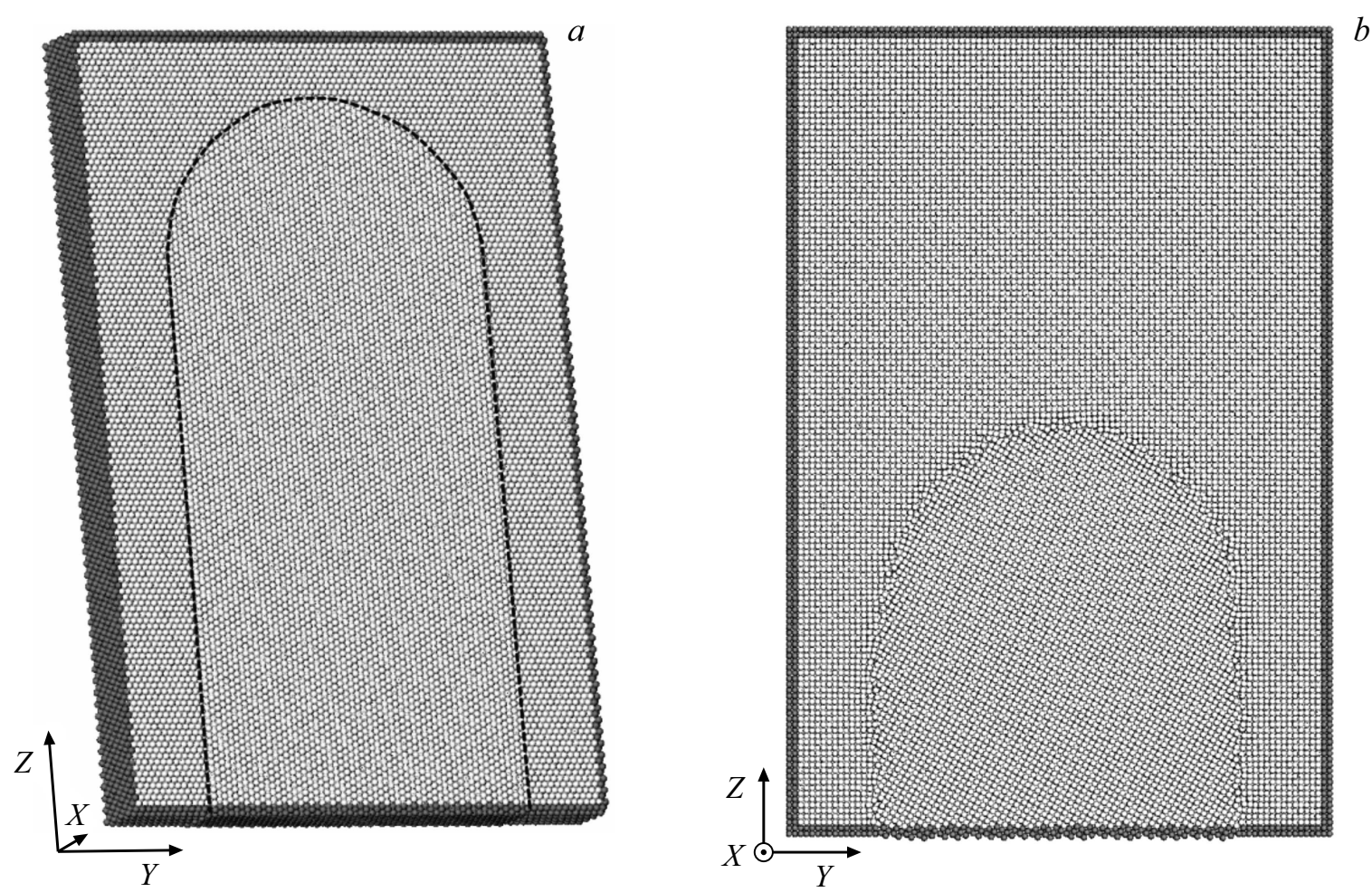

Рис. 1. Расчетная ячейка для моделирования миграции границы наклона в никеле: $a$ - стартовая структура ячейки с границей $\langle 111\rangle 30^{\circ} ; b-$ ячейка с границей $\langle 100\rangle 30^{\circ}$ после моделирования миграции в течение 650 рs при температуре $1700 \mathrm{~K}$ (изображение получено после охлаждения ячейки). Темно-серые атомы на краю расчетной ячейки в процессе компьютерного эксперимента оставались неподвижными (жесткие граничные условия).

метод позволяет проводить моделирование миграции границ зерен с четко заданными кристаллографическими параметрами и конкретной концентрацией вакансий на начальном этапе. Преимуществом модели является то, что при постоянной температуре объем расчетной ячейки оставался постоянным, как и вводимый в расчетную ячейку на начальном этапе дополнительный свободный объем. Большеугловые границы наклона $\langle 111\rangle$ и $\langle 100\rangle$ были выбраны в связи с их сравнительно высокой подвижностью, позволяющей проводить измерения скорости их миграции в молекулярно-динамических экспериментах [16-18]. Исследования проводились на примере никеля - типичного и хорошо изученного ГЦК металла, имеющего большое прикладное значение. Ранее с использованием аналогичной модели нами уже были проведены исследования влияния на скорость миграции границ наклона $\langle 111\rangle$ и $\langle 100\rangle$ в никеле упругой деформации [19] и примесей легких элементов [6], где было показано, что наличие примесей, как и упругое сжатие, приводят к снижению скорости миграции.

\section{2. Описание модели}

Взаимодействия атомов металла друг с другом описывались многочастичными потенциалами КлериРосато [20], построенными в рамках модели сильной связи. Потенциальная энергия $i$-го атома в этом случае находится с помощью выражения

$$
\begin{aligned}
U_{i}= & \sum_{j} A \exp \left(-p\left(\frac{r_{i j}}{r_{0}}-1\right)\right) \\
& -\sqrt{\sum_{j} \xi^{2} \exp \left(-2 q\left(\frac{r_{i j}}{r_{0}}-1\right)\right)} .
\end{aligned}
$$

Здесь $A, p, q, \xi, r_{0}$ - параметры потенциала; $r_{i j}-$ расстояние между $i$-м и $j$-м атомами. Потенциалы данного типа неоднократно использовались в молекулярнодинамических моделях и прошли апробацию по большому числу характеристик [21,22]. Опыт их применения показывает, что с их помощью удается описать разнообразные свойства металлов и сплавов.

За основу модели миграции границы зерен была взята методика, предложенная и развитая в работах $[1,4,23,24]$. Согласно данной модели, создается четко аттестованная граница в форме полупетли, как на рис. 1, $a$ (показана черной пунктирной линией). Сила натяжения границы, которая, подобно поверхностному натяжению, возникает вследствие стремления границы минимизировать свою энергию, является причиной направленного перемещения границы в сторону уменьшения ее площади. Сила, провоцирующая миграцию, зависит от энергии границы, 
которая в свою очередь зависит от угла разориентации (в связи с этим, в частности, большеугловые границы мигрируют быстрее малоугловых $[1,2])$. Скорость перемещения границы в результате действия этой силы зависит, помимо самой силы, еще от кривизны и подвижности границы. Влияние ширины полупетли (кривизны границы) подробно проанализировано, например, в [24]. В нашей модели, как и в работах $[23,24]$, кривизна создавалась относительно высокой для того, чтобы скорость миграции была достаточной для того, чтобы ее можно было измерить методом молекулярной динамики.

Сила и скорость миграции при постоянной температуре оставались в рассматриваемой модели приблизительно постоянными в течение почти всего движения границы, плавно уменьшаясь к концу компьютерного эксперимента. При этом форма верхней части полупетли в большинстве случаев почти не изменялась, за исключением случаев задержки границы дефектами структуры. На рис. $1, b$ изображен пример изменения положения границы зерен в результате ее миграции в течение молекулярно-динамического эксперимента.

Расчетная ячейка содержала примерно 120000 атомов и имела высоту $28.5 \mathrm{~nm}$, ширину $19.0 \mathrm{~nm}$ и толщину $2.4 \mathrm{~nm}$. Вдоль оси $X$ (рис. 1) имитировалось бесконечное повторение структуры, т.е. были наложены периодические граничные условия. На краю расчетной ячейки границы зерен должны быть зафиксированы, что подразумевает сохранение ориентации кристаллической решетки двух разных зерен на границе ячейки. В связи с этим по осям $Y$ и $Z$ границы ячейки были жестко закреплены для фиксации заданной разориентации зерен: атомы, окрашенные в темно-серый цвет на рис. 1, оставались неподвижными в процессе компьютерного эксперимента.

Исследования были проведены для границ наклона $\langle 111\rangle$ и $\langle 100\rangle$ с двумя углами разориентации: 20 и $30^{\circ}$. После создания расчетных ячеек, содержащих границу зерен, проводилась первичная структурная релаксация с возможностью изменения объема ячейки для достижения нулевого общего давления. Это было необходимо для получения структуры, соответствующей нулевой начальной концентрации вакансий.

Шаг интегрирования по времени в методе молекулярной динамики был равен 2 fs. Температура в модели задавалась через начальные скорости атомов согласно распределению Максвелла-Больцмана, при этом учитывалось изменение параметра решетки вследствие теплового расширения. Для сохранения температуры постоянной в процессе моделирования использовался термостат Нозе-Гувера. Моделирование миграции границ проводилось при температуре $1700 \mathrm{~K}$. Выбор температуры, почти равной температуре плавления никеля, обусловлен тем, что при данной температуре скорость миграции границ наибольшая.

Свободный объем вводился в модели путем задания определенной концентрации вакансий. В основных компьютерных экспериментах объем расчетной ячейки после задания температуры оставался постоянным, что позволяло четко оценивать величину свободного объема в ячейке, несмотря на процессы абсорбции вакансий границей и объединение вакансий друг с другом с образованием вакансионных кластеров. Концентрация вводимых вакансий в расчетную ячейку на начальном этапе моделирования варьировалась в широком диапазоне от 0 до 7\%. Последнее значение более, чем на два порядка превышает равновесную концентрацию вакансий, но, вместе с тем, это позволяет рассмотреть влияние вакансионных кластеров, возникающих, например, при радиационном повреждении, на подвижность границ. Суммарный дополнительный свободный объем в расчетной ячейке складывался, таким образом, из вакансионного и свободного объема, содержащегося изначально в границе зерен $(1,2 \%$ от всего объема расчетной ячейки).

\section{3. Результаты и обсуждение}

На рис. 2 изображены зависимости скорости миграции рассматриваемых границ зерен при температуре $1700 \mathrm{~K}$
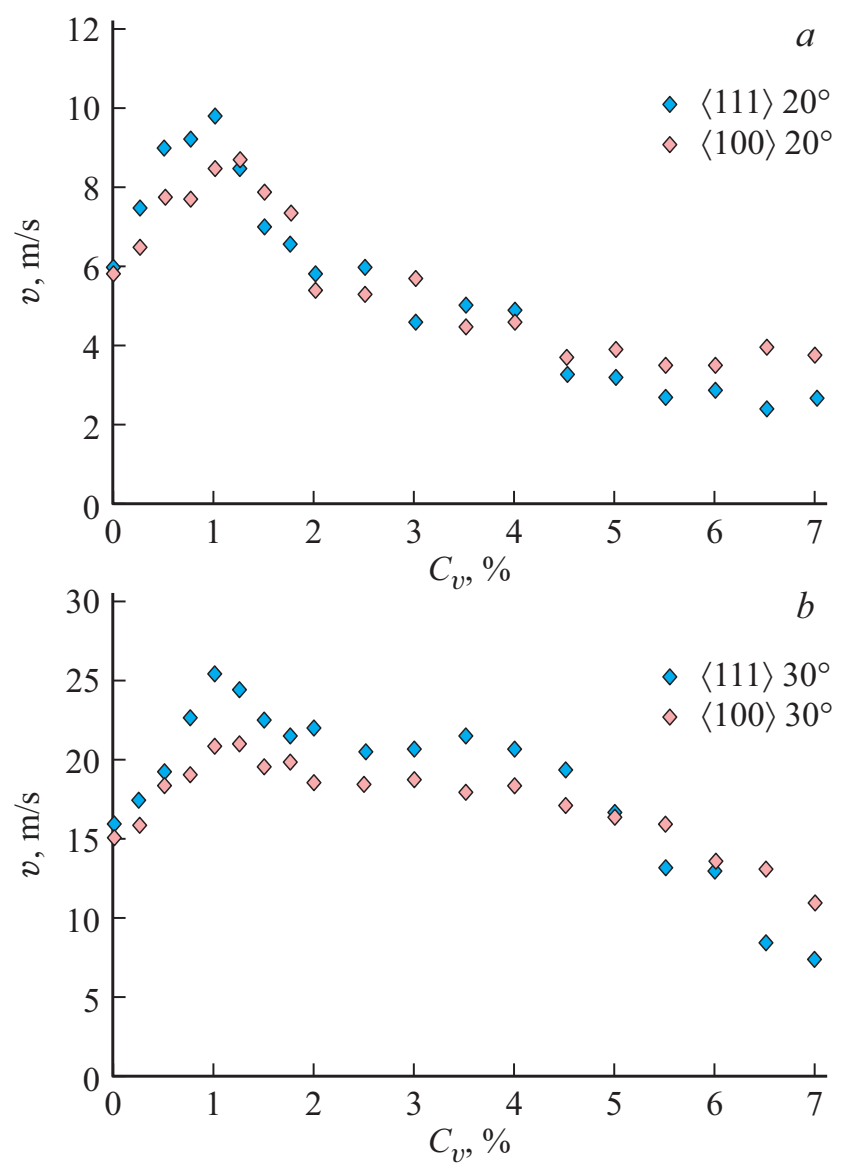

Рис. 2. Зависимости скорости миграции границ наклона $\langle 111\rangle$ и $\langle 100\rangle$ при температуре $1700 \mathrm{~K}$ в никеле от начальной концентрации вакансий в расчетной ячейке: $a-$ с углом разориентации $20^{\circ} ; b-$ с углом разориентации $30^{\circ}$. 

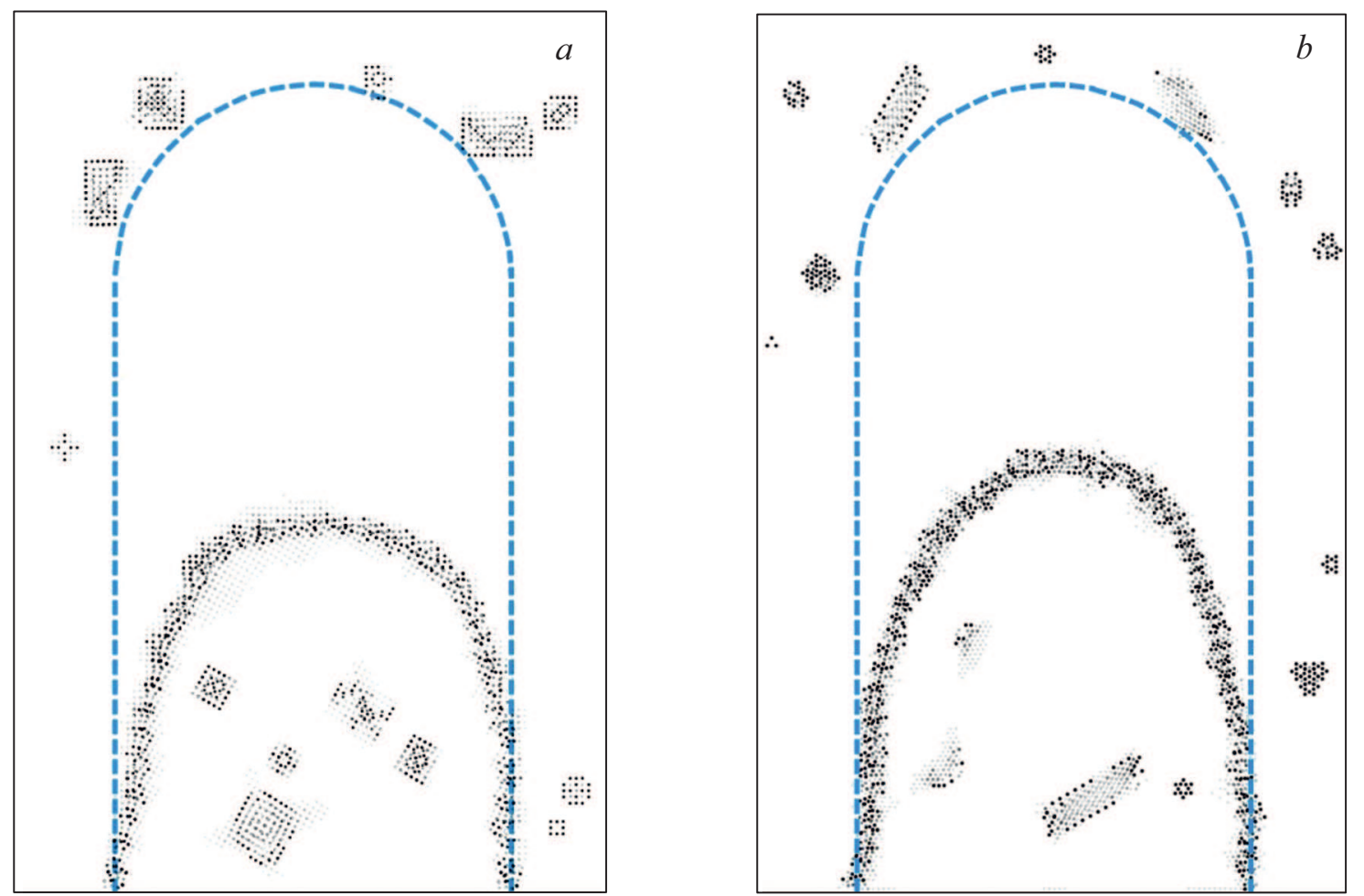

Рис. 3. Примеры поглощения вакансий и вакансионных кластеров мигрирующей границей зерен: $a-$ моделирование миграции границы $\langle 100\rangle 30^{\circ}$ при начальной концентрации вакансий $1 \%$ в течение $700 \mathrm{ps} ; b-$ моделирование миграции границы $\langle 111\rangle 30^{\circ}$ при начальной концентрации вакансий $0.75 \%$ в течение 500 ps. Изображения получены после охлаждения структуры расчетной ячейки. Штриховой линией показано начальное положение границы.

от концентрации вакансий, введенных на начальном этапе компьютерного эксперимента. Скорость миграции оценивалась по смещению верхней части границы в форме полупетли (рис. 1) вдоль оси Z. Столь высокие значения скорости (от 2 до $26 \mathrm{~m} / \mathrm{s}$ ) характерны для молекулярно-динамического моделирования миграции границ зерен (это необходимо для прямого измерения скорости миграции) [18,23-26] и достигнуты в данном случае благодаря сочетанию трех факторов: высокой изначальной кривизны границы, высокой температуры, близкой к температуре плавления, и высокой подвижности рассматриваемых границ.

Как видно, все рассмотренные зависимости скорости миграции границ зерен от свободного объема немонотонны и имеют четко выраженный максимум вблизи 1\% введенных на начальном этапе вакансий. Рост скорости миграции границ по мере увеличения концентрации вакансий при сравнительно невысоких их концентрациях согласуется с косвенными экспериментальными данными $[7,8]$. Как говорилось во введении, это связано с повышением диффузионной подвижности атомов на границе зерен вследствие увеличения свободного объема. Перемещения атомов в области границы облегчены при наличии дополнительного свободного пространства свободного объема, на что косвенно указывает также, например, влияние на скорость миграции гидростатического сжатия [19,27].
Из сравнения зависимостей, полученных для границ с осями разориентации $\langle 111\rangle$ и $\langle 100\rangle$ видно, что они мигрируют с примерно одной скоростью в рассматриваемой модели. В [18] нами подчеркивалось, что это касается только большеугловых границ. По мнению многих авторов, для большого класса большеугловых границ и границ смешанного типа энергия приблизительно одинакова, в связи с чем, например, большинство углов между границами в тройных стыках в поликристаллах близки к $120^{\circ}[28,29]$. Вместе с тем, скорость миграции малоугловых границ $\langle 111\rangle$ и $\langle 100\rangle$, как было показано нами в [18], сильно отличается - при малых углах разориентации границы $\langle 100\rangle$ мигрируют почти в два раза медленнее границ $\langle 111\rangle$.

При концентрациях вакансий примерно до 3\% характерным явлением было поглощение мигрирующей границей всех встречаемых на пути вакансий и вакансионных кластеров, в результате чего кристалл позади движущейся границы очищался от дефектов. На рис. 3 с помощью визуализатора среднего расстояния до ближайших атомов показаны примеры подобного „очищения“ при миграции границ $\langle 100\rangle$ и $\langle 111\rangle$ при начальной концентрации вакансий 1 и $0.75 \%$ соответственно. Данный визуализатор дает представление о распределении свободного объема. Для каждого атома проводился расчет среднего расстояния до ближайших атомов. Если среднее расстояние незначительно отличалось от рассто- 

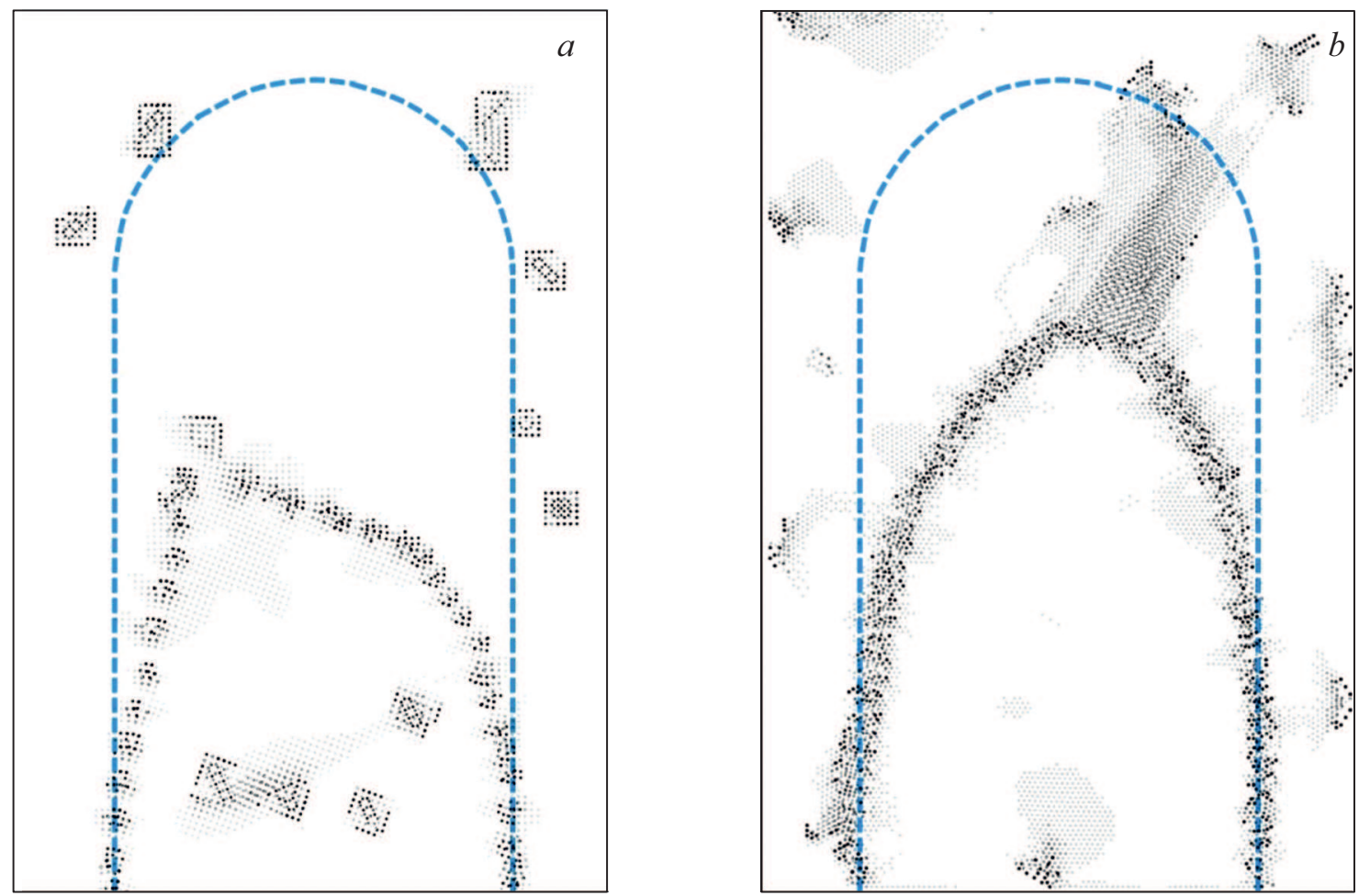

Рис. 4. Примеры торможения миграции границ зерен вакансионными кластерами: $a-$ моделирование миграции границы $\langle 100\rangle$ $20^{\circ}$ при начальной концентрации вакансий $1.5 \%$ в течение $1700 \mathrm{ps} ; b-$ моделирование миграции границы $\langle 111\rangle 30^{\circ}$ при начальной концентрации вакансий $5 \%$ в течение 500 ps. Изображения получены после охлаждения структуры расчетной ячейки. Штриховой линией показано начальное положение границы.

яния, соответствующего идеальному кристаллу, атом не изображался. В противном случае атом закрашивался в тот или иной оттенок серого цвета (чем темнее, тем больше свободного объема вблизи данного атома).

При дальнейшем повышении начальной концентрации вакансий более $1 \%$ миграция рассматриваемых границ, напротив, замедлялась, а при концентрациях выше примерно 4\% начинала падать еще сильнее (рис. 2). Используемый нами визуализатор свободного объема позволял ясно увидеть причину такого поведения. Как оказалось, при сравнительно высоких начальных концентрациях вакансий граница утрачивала способность поглощать все встречающиеся на пути вакансионные кластеры. Они прикреплялись к ней, и, будучи гораздо менее подвижными, эффективно тормозили ее миграцию (рис. 4). Большие вакансионные кластеры в этом случае начинали работать не как источники свободного объема, а как и большинство дефектов или инородных частиц, которые при сочетании положительной энергии связи с границей зерен и слабой диффузионной подвижности приводят к снижению скорости миграции границы [1-3].

На рис. 4 изображены примеры задержки границы вакансионным кластером. Вакансионные кластеры, которые наблюдались в модели, представляли собой, как правило, тетраэдры дефектов упаковки, часто неидеальные и сопряженные друг с другом (рис. 4,a) (в процессе дальнейшей структурной трансформации при высоких температурах они, как правило, перестраивались в один дефект) или дислокационные петли (рис. 4, $b$ ).

Второй причиной снижения скорости миграции границ являлось уменьшение движущей силы, которая обычно считается не зависящей от концентрации вакансий при сравнительно низких ее значениях [2,7]. Но дело в том, что, как было отмечено в [14,15], границы обладают не бесконечной сорбционной способностью по отношению к вакансиям. При чрезмерно большой величине сорбированного границей свободного объема, наступает момент, когда сокращение ее площади приводит к частичной эмиссии избыточного свободного объема обратно в кристалл. Поверхностное натяжение границы зерен и, соответственно, движущая сила миграции в таком случае снижаются.

\section{4. Заключение}

Методом молекулярной динамики проведено исследование влияния концентрации вакансий на скорость миграции большеугловых границ наклона с осями разориентации $\langle 111\rangle$ и $\langle 100\rangle$ в никеле. При повышении концентрации введенных на начальном этапе моделирования вакансий до $1 \%$ скорость миграции границ увеличивалась, что было обусловлено облегчением диффузионных перемещений атомов вследствие появления 
дополнительного свободного пространства в границе. Однако при дальнейшем повышении свободного объема скорость миграции границ снижалась, а при концентрациях вакансий выше примерно 4\% падала еще сильнее. Было выяснено, что при сравнительно высоких начальных концентрациях вакансий граница утрачивала способность поглощать все встречающиеся на пути вакансионные кластеры. Они прикреплялись к ней, и, будучи значительно менее подвижными, эффективно тормозили ее миграцию. Второй причиной снижения скорости миграции границ являлось уменьшение их поверхностного натяжения и, соответственно, движущей силы вследствие конечной сорбционной способности границ по отношению к вакансиям - при чрезмерно большой величине сорбированного границей свободного объема, наступал момент, когда сокращение ее площади уже приводило к частичной эмиссии избыточного свободного объема обратно в кристалл.

\section{Конфликт интересов}

Авторы заявляют, что у них нет конфликта интересов.

\section{Список литературы}

[1] G. Gottstein, L.S. Shvindlerman. Grain Boundary Migration in Metals: Thermodynamics, Kinetics, Applications. 2 nd ed. CRC Press, Boca Raton (2009). 711 p.

[2] О.А. Кайбышев, Р.3. Валиев. Границы зерен и свойства металлов. Металлургия, М. (1987). 216 с.

[3] Q. Zhu, S.C. Zhao, C. Deng, X.H. An, K.X. Song, S.X. Mao, J.W. Wang. Acta Mater. 199, 42 (2020).

[4] G. Gottstein, D.A. Molodov, L.S. Shvindlerman. Interface Sci. 6, 7 (1998).

[5] L.-L. Niu, Q. Peng, F. Gao, Zh. Chen, Y. Zhang, G.-H. Lu. J. Nucl. Mater. 512, 246 (2018).

[6] G.M. Poletaev, I.V. Zorya, R.Y. Rakitin, M.A. Iliina, M.D. Starostenkov. Lett. Mater. 9, 4, 391 (2019).

[7] F. Haessner. J. Phys. Colloq. 36, C4, 345 (1975).

[8] H. Takahashi, N. Hashimoto. Mater. Trans. 34, 11, 1027 (1993).

[9] G. Lu, N. Kioussis. Phys. Rev. B 64, 024101 (2001).

[10] P. Ballo, N. Kioussis, G. Lu. Phys. Rev. B 64, 024104 (2001).

[11] B. Oberdorfer, D. Setman, E.-M. Steyskal, A. Hohenwater, W. Sprengel, M. Zehetbauer, R. Pippan, R. Wurschum. Acta Mater. 68, 189 (2014).

[12] S.V. Divinski. Diffusion Foundations 5, 57 (2015).

[13] Р.Т. Мурзаев, А.А. Назаров. Физика металлов и металловедение 102, 2, 214 (2006). [R.T. Murzaev, A.A. Nazarov. Phys. Met. Metallography 102, 2, 198 (2006).]

[14] Y. Estrin, G. Gottstein, E. Rabkin, L.S. Shvindlerman. Acta Mater. 49, 673 (2001).

[15] V.G. Sursaeva, G. Gottstein, L.S. Shvindlerman. Scr. Mater. 116, 91 (2016).

[16] Y. Huang, F.J. Humphreys. Acta Mater. 47, 2259 (1999).

[17] Y. Huang, F.J. Humphreys. Mater. Chem. Phys. 132, 166 (2012).
[18] Г.М. Полетаев, И.В. Зоря, М.Д. Старостенков, Р.Ю. Ракитин, П.Я. Табаков. ЖЭТФ 155, 1, 96 (2019). [G.M. Poletaev, I.V. Zorya, M.D. Starostenkov, R.Yu. Rakitin, P.Ya. Tabakov. J. Exp. Theor. Phys. 128, 1, 88 (2019)].

[19] Г.М. Полетаев, И.В. Зоря, Р.Ю. Ракитин, Д.В. Коханенко, М.Д. Старостенков. Изв. вузов. Черная металлургия 61, 12, 974 (2018).

[20] F. Cleri, V. Rosato. Phys. Rev. B 48, 1, 22 (1993).

[21] Г.М. Полетаев, И.В. Зоря. ЖЭТФ 158, 3, 485 (2020). [G.M. Poletaev, I.V. Zorya. J. Exp. Theor. Phys. 131, 3, 432 (2020)].

[22] Г.М. Полетаев, Д.В. Новоселова, И.В. Зоря, М.Д. Старостенков. ФТТ 60, 5, 846 (2018). [G.M. Poletaev, D.V. Novoselova, I.V. Zorya, M.D. Starostenkov. Phys. Solid State 60, 5, 847 (2018)].

[23] M. Upmanyu, D.J. Srolovitz, L.S. Shvindlerman, G. Gottstein. Acta Mater. 50, 1405 (2002).

[24] H. Zhang, M. Upmanyu, D.J. Srolovitz. Acta Mater. 53, 79 (2005).

[25] N. Bernstein. Acta Mater. 56, 1106 (2008).

[26] Z.T. Trautt, Y. Mishin. Acta Mater. 60, 2407 (2012).

[27] D.A. Molodov, B.B. Straumal, L.S. Shvindlerman. Scr. Mater. 18, 3, 207 (1984).

[28] M.A. Fortes, A.M. Deus. Materials Science Forum 455-456, 648 (2004).

[29] O.B. Perevalova, E.V. Konovalova, N.A. Koneva, E.V. Kozlov. J. Mater. Sci. Technol. 19, 6, 593 (2003).

Редактор Ю.Э. Китаев 